Marquette University

e-Publications@Marquette

Mechanical Engineering Faculty Research and

Publications

Mechanical Engineering, Department of

2019

An Efficient Monte Carlo-Based Solver for Thermal Radiation in

Participating Media

Joseph Farmer

Somesh Roy

Follow this and additional works at: https://epublications.marquette.edu/mechengin_fac

Part of the Mechanical Engineering Commons 


\title{
An Efficient Monte Carlo-Based Solver for Thermal Radiation in Participating Media
}

\author{
Joseph A. Farmer \& Somesh P. Roy
}

Department of Mechanical Engineering, Marquette University, Milwaukee, WI, USA.

\begin{abstract}
Monte Carlo-based solvers, while well-suited for accurate calculation of complex thermal radiation transport problems in participating media, are often deemed computationally unattractive for use in the solution of real-world problems. The main disadvantage of Monte Carlo (MC) solvers is their slow convergence rate and relatively high computational cost. This work presents a novel approach based on a low-discrepancy sequence (LDS) and is proposed for reducing the error bound of a Monte Carlo-based radiation solver. Sobols sequence - an LDS generated with a bit-by-bit exclusive-or operator - is used to develop a quasi-Monte Carlo (QMC) solver for thermal radiation in this work. Preliminary results for simple radiation problems in participating media show that the QMC-based solver has a lower error than the conventional MC-based solver. At the same time, QMC does not add any significant computational overhead. This essentially leads to a lower computational cost to achieve similar error levels from the QMC-based solver than the MC-based solver for thermal radiation.
\end{abstract}

\section{KEY WORDS}

Radiation, Combustion, Monte Carlo, Quasi Monte Carlo, Low Discrepancy Sequence

\section{INTRODUCTION}

Radiation is an important mode of heat transfer particularly in high temperature systems such as in combustion. Radiative heat transfer via participating media is a highly nonlocal process with strong nonlinear effects. It is modeled using the radiative transfer equation (RTE) [1]

(1)

$$
\frac{d I_{\eta}}{d s}=\hat{s} \bullet \nabla I_{\eta}=\kappa_{\eta} I_{b \eta}-\beta_{\eta} I_{\eta}+\frac{\sigma_{s \eta}}{4 \pi} \int_{4 \pi} I_{\eta}(\hat{s}) \Phi_{\eta}\left(\hat{s}_{i}, \hat{s}\right) d \Omega_{i},
$$

where $\eta$ represents wavenumber indicating the RTE is unique for each wavenumber. Equation 1 is a fivedimensional, integro-differential equation. The difficulty of solving the RTE has led to various approximate solvers for different applications. For instance, the method of spherical harmonics $\left(P_{N}\right.$ method) was introduced by J.H. Jeans [2] as a method of arbitrary order to solve the RTE in relation to radiation emitted from stars. The $P_{N}$ method was adapted for combustion-related applications later by Modest and co-workers [e.g., 3-7, etc.]. The method of discrete ordinates ( $S_{N}$ method), first introduced by S. Chandrasekhar [8], was proposed to solve RTE in relation to atmospheric radiation and was further developed by several researchers [e.g., 9-11, etc.]. The $P_{N}$ and $S_{N}$ methods both implement different 
techniques to deconstruct the RTE into a set of partial differential equations. Other methods include the zonal method introduced by Hottel [12], the moment method introduced by Eddington [13], etc. A detailed review of various methods can be found in various literature including $[1,10,14-16]$.

The $S_{N}$ and $P_{N}$ methods are commonly chosen for combustion simulations. For development and detailed use of these and other RTE solvers in combustion applications, the reader is referred to articles such as $[1,9,14,17$, and references therein]. However, these solvers have their limitations. For example, the $S_{N}$ method suffers from ray effects and false scattering [1]. The $P_{N}$ method converges slowly and is computationally expensive $[17,18]$. Additionally, the approximate solvers are often limited to simple geometries and boundary conditions.

Alternatively, a statistical Monte Carlo-based solver can produce the exact solution of the RTE irrespective of the complexity of the problem $[1,9]$. However, for accurate solutions, Monte Carlo (MC) solvers require a statistically large number of samples which contributes to the high computational cost. For this reason, the use of Monte Carlo-based solvers remains limited. In this work, a novel scheme using low-discrepancy sequences (LDS) is proposed to increase the efficiency of a Monte Carlo-based thermal radiation solver for participating media. While LDS have been used in Monte Carlo-based solvers in the past, their use to solve radiation-related problems is mostly restricted to surface transfer [e.g., 19, 20, etc.]. The next section discusses the Monte Carlo method and LDS and the results obtained for one-dimensional plane parallel media cases. Finally the advantages of QMC are discussed in the conclusion.

\section{EFFICIENT (QUASI) MONTE CARLO METHOD}

The solution of radiative transfer via the Monte Carlo method is achieved by emitting and tracking a statistically large number of "rays" or "photon bundles". A ray can be thought of as a bundle of photons with a finite amount of energy propagating through and interacting with the participating media. Each ray is characterized by its origin $(x ; y ; z)$, direction $(\theta ; \phi)$, wavenumber $\eta$, and an associated energy content. The first six variables for each ray are determined by sampling six uniformly distributed random numbers $\left(R_{x} ; R_{y} ; R_{z} ; R_{\theta} ; R_{\phi}\right.$, and $\left.R_{\eta}\right)$ as outlined in [1, 21-23]. The accuracy of the method can be estimated by the statistical error, usually represented by the standard deviation of an evaluated variable over many iterations. Theoretically, with $N$ samples (in this case, $N$ rays), the error limit scales as $O\left(N^{-0: 5}\right)$, whereas the computational cost usually increases linearly with number of rays.

In this work, we propose to use a deterministic low-discrepancy sequence, instead of uniformly distributed random numbers in the conventional MC. This leads to a quasi-Monte Carlo (QMC) solver for radiation. Often random numbers for a Monte Carlo solution are generated using pseudo-random number generators [e.g., see 24], which follow deterministic algorithms, but still satisfy the statistical properties of a uniform random distribution reasonably well. Low discrepancy sequences are, on the other hand, deterministic sequences designed to produce equidistant points in the sequence [25]. By their nature, LDS can produce a low-variance distribution at the cost of true randomness. Because the samples are not random, the Monte Carlo approach using LDS is conventionally referred as quasi-Monte Carlo (QMC) [25]. These methods have been in development for many years and are extensively used in financial modeling [26]. A review of low-discrepancy sequences and QMC can be found in [25]. In general, QMC has shown promise in solving high-dimensional problems. For this study, Sobol's sequence was chosen as the LDS of interest based on an empirical study of the computational efficiency of the 
sequence following the recommendations of [27, 28]. For details of conventional Monte Carlo solvers [1] and Sobol's sequence $[29,30]$ the reader is directed to the respective literature. As the first attempt to use Sobol's sequence in QMC to solve the RTE, the six random numbers required in the standard MC method $\left(R_{x} ; R_{y} ; R_{z} ; R_{\theta} ; R_{\phi}\right.$, and $\left.R_{\eta}\right)$ are replaced by six samples from Sobol sequences generated with different primitive polynomials (a six-dimensional Sobol sequence).

\section{RESULTS AND DISCUSSION}

\subsection{Target configurations}

In this work we present a proof-of-concept for the benefits of QMC using a Sobol's sequence over conventional MC solvers for one-dimensional problems. Results from both $\mathrm{MC}$ and $\mathrm{QMC}$ solver are compared with analytical solutions of one-dimensional, plane-parallel media under seven different configurations. Out of the seven conditions shown here, five are gray medium and two are nongray. The cases are listed in Table 1. The comparison is made based on the divergence of radiative heat flux, $\nabla \cdot$ $Q$.

Table 1 Target cases (one-dimensional plane-parallel medium)

\begin{tabular}{|l|l|l|l|}
\hline Case & Absorption Coefficient $\left(\mathrm{m}^{-1}\right)$ & Medium Temperature $(\mathrm{K})$ & Wall Temperature $(\mathrm{K})$ \\
\hline 1 & 10 & 1200 & 800 \\
\hline 2 & 100 & 1200 & 800 \\
\hline 3 & 1 & 1200 & 800 \\
\hline 4 & $1-100$ & $1680-1200$ & 800 \\
\hline 5 & $20-80$ & $1200-2200$ & 800 \\
\hline 6 & Nongray & 2000 & Cold (non-emitting) \\
\hline 7 & Nongray & $2100-2700$ & Cold (non-emitting) \\
\hline
\end{tabular}

In all seven cases the distance between the plates are kept at $0.1 \mathrm{~m}$ while the absorption coefficient and temperature of the medium are varied. In the first three cases (Case 1, 2, and 3), both the absorption coefficient and the medium temperature are kept uniform. In Case 4, both absorption coefficient and medium temperature are linearly varied (in opposite directions) between plates. Case 5 contains a square-wave-like distribution with step changes in both absorption coefficient and medium temperature (around $0.02 \mathrm{~m}$ from both plates). In Cases $1-5$ the walls are assumed to be black and emitting at 800 $\mathrm{K}$. The last two cases contain nongray medium between the plates. We chose a uniformly distributed $\mathrm{CO} 2$ (mole fraction 0.2) for the radiatively participating gas in the medium. In Case 6, temperature distribution is also assumed uniform at $2000 \mathrm{~K}$. In Case 7, temperature distribution is varied (somewhat arbitrarily) to achieve a condition with relatively sharp gradients in the medium. Walls for Case 6 and 7 are assumed to be cold (non-emitting) and black.

\subsection{Accuracy and error estimation}

The accuracy of each method is shown in terms of distribution of relative error, as well as variation of average relative errors with number of rays used in each simulation. The relative error $(\varepsilon)$ is computed at each computational cell $i$ by

(2) 


$$
\varepsilon_{i, M C}=\frac{1}{N} \sum_{j}^{N}\left|\frac{(\nabla \bullet Q)_{i, \text { analytical }}-(\nabla \bullet Q)_{i, j, M C}}{(\nabla \bullet Q)_{i, \text { analytical }}}\right|,
$$

(3)

$$
\varepsilon_{i, Q M C}=\left|\frac{(\nabla \bullet Q)_{i, \text { analytical }}-(\nabla \bullet Q)_{i, Q M C}}{(\nabla \bullet Q)_{i, \text { analytical }}}\right|,
$$

where $N$ is the number of statistical runs of MC. Because of its deterministic nature, only one instance of QMC simulation was sufficient for each configuration. The convergence rate is represented as the decrease in the spatially-averaged relative error with number of rays used in respective simulations. The spatially-averaged relative error is calculated as the arithmetic mean of relative errors over all computational cells.

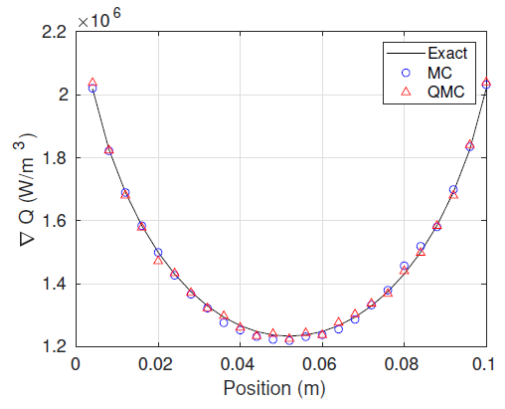

(a) Case 1

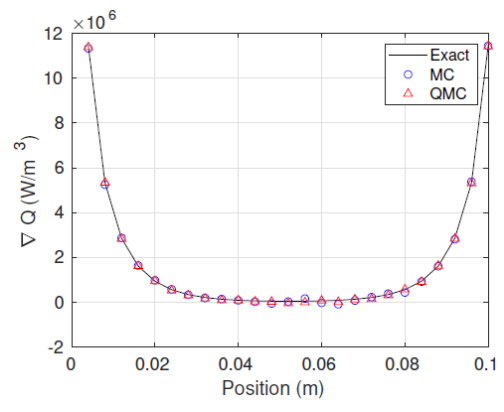

(b) Case 2

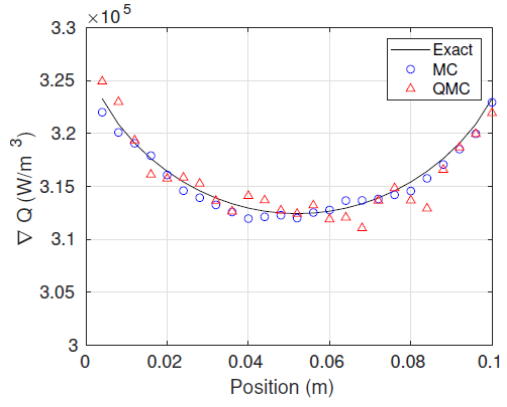

(c) Case 3

Fig. 1 Comparison of $\nabla \bullet Q$ (a) Case 1 with 20,000 rays, (b) Case 2 with 120,000 rays, and (c) Case 3 with 20,000 rays

\section{Cases 1, 2, and 3-uniform medium}

Figure 1 presents the results from analytical, MC, and QMC solution for Cases 1 using 20,000 rays, Case 2 using 120,000 rays, and Case 3 using 20,000 rays. Since Case 2 is optically thicker than Case 1 and 3 , more rays were needed for this case to get a reasonably good solution. This is an expected phenomenon in Monte Carlo solution of optically thick media. Both MC and QMC show reasonable agreement with the analytical solution. The advantage of QMC over $\mathrm{MC}$ becomes more apparent in Fig. 2, where relative errors are shown for the three cases. For Case 1 and 2 the relative error from QMC is noticeably lower than $\mathrm{MC}$ for the same number of rays (Fig. $2 \mathrm{a}$ and $2 \mathrm{c}$ ). For the optically thick Case 2 , the errors at the center of the plates are very high (as expected from a Monte Carlo simulation for optically thick uniform medium). For the optically thin Case 3, there is no distinguishable advantage of QMC over MC (Fig. 2e). The convergence rate plots (Fig, $2 b$ and $2 \mathrm{~d}$ and $2 \mathrm{f}$ ) show similar convergence rate for $\mathrm{MC}$ and $\mathrm{QMC}$ for Case 1 and 3. For the optically thick case, however, the convergence rate of standard MC is faster than QMC. While the relative convergence rate does not show any significant improvement for QMC, the actual error of QMC is at least a factor of two lower than MC. It is expected that with more rays the results from MC simulation will achieve similar or better results than QMC. However, increasing the number of rays will also increase the computational cost. This indicated that the QMC is advantageous in these configurations. 
Case 4 and 5 - non-uniform gray medium:

Figure 3 presents the results from the analytical, MC, and QMC solution for Case 4. Both MC and QMC show agreement with the analytical solution. The advantage of QMC over MC is more apparent when comparing the relative error of each method (Fig. 3b and 3c). Similar to Cases 1-3, in optically thin regions there is little distinguishable advantage for the QMC method. In optically thick regions, relative error of the QMC method is, again, noticeably lower. The convergence rate plot (Fig. 3c) shows similar convergence rate; however, the actual error of QMC is again at least a factor of two smaller than MC. The same trends can be seen for Case 5 as presented in Fig. 4

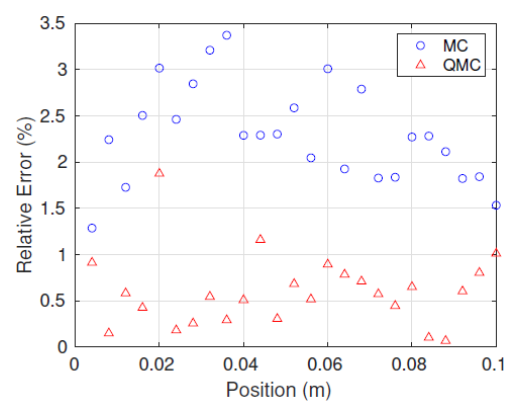

(a) Relative error for Case 1 with 20,000 rays

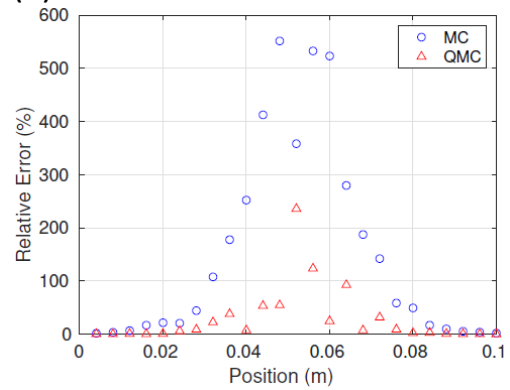

(c) Relative error for Case 2 with 120,000 rays

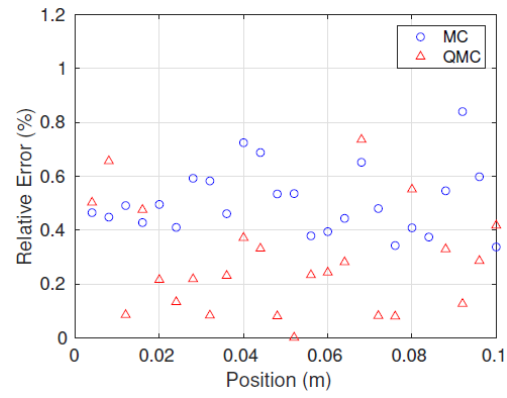

(e) Relative error for Case 3 with 20,000 rays

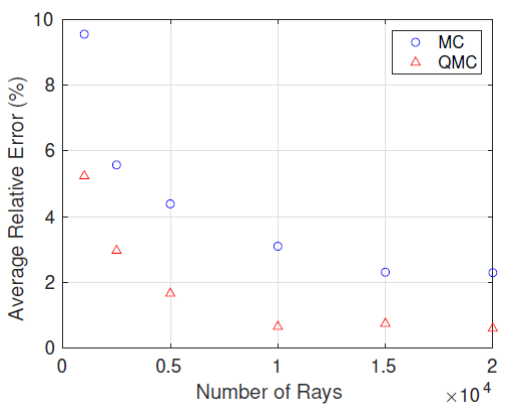

(b) Convergence rate for Case 1

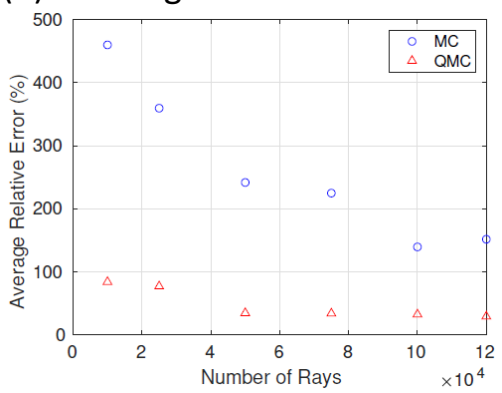

(d) Convergence rate for Case 2

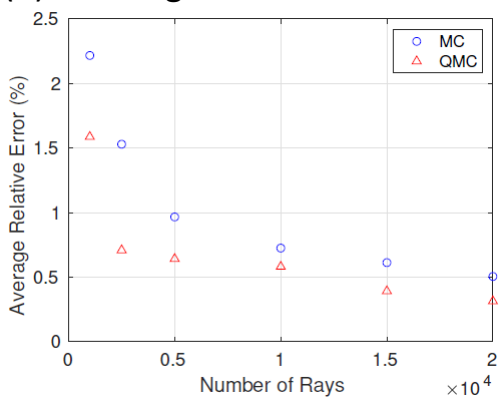

(f) Convergence rate for Case 3

Fig. 2 Relative errors and convergence rates for Cases 1, 2, and 3 


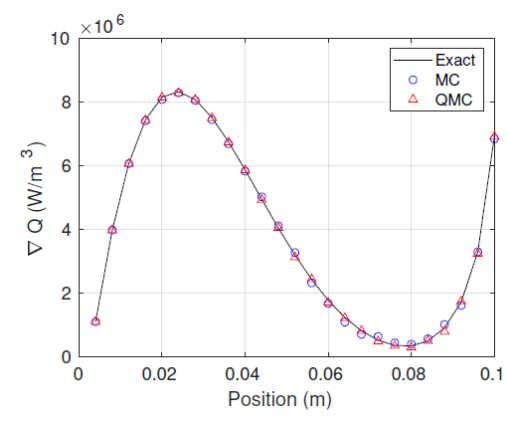

(a) Comparison of $\nabla \bullet Q$ with 75,000 rays

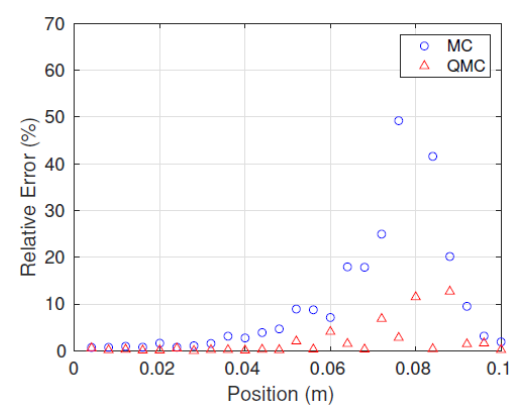

(b) Relative error with 75,000 rays

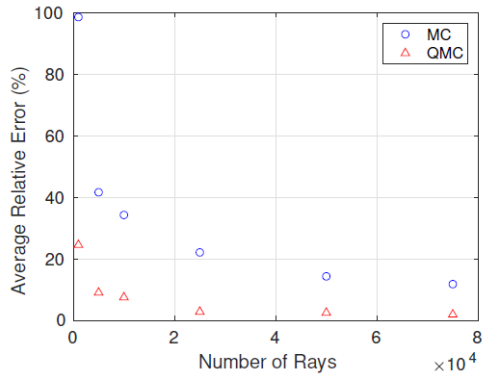

(c) Convergence rates

Fig. 3 Accuracy and convergence rates for Case 4

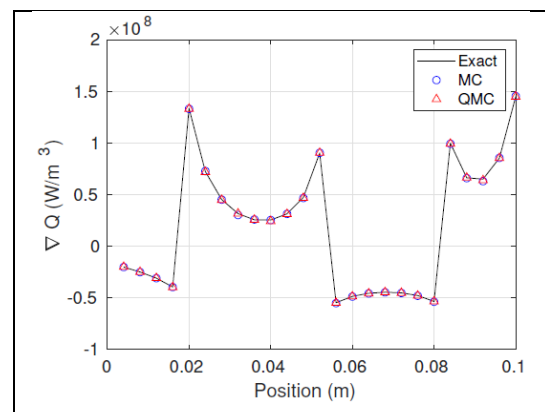

(a) Comparison of $\nabla \cdot Q$ with 75,000 rays

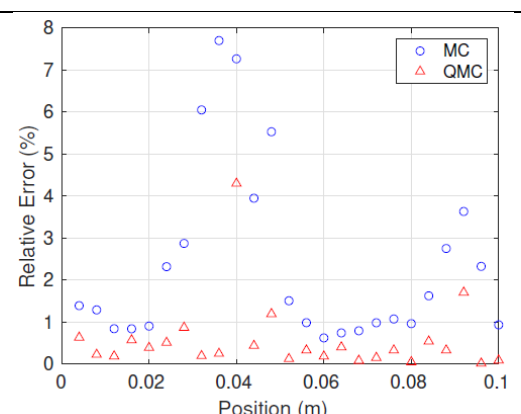

(b) Relative error with 75,000 rays

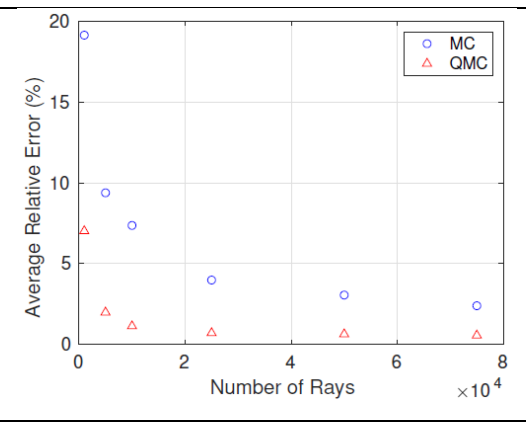

(c) Convergence rates

Fig. 4 Accuracy and convergence rates for Case 5

Case 6 and 7 - Nongray medium:

The nongray medium constitutes a uniform distribution of $\mathrm{CO} 2$. Nongray radiative properties of $\mathrm{CO} 2$ are calculated from a line-by-line database obtained from the HITEMP spectroscopic database [31]. In Case 6 , the medium temperature is at uniform $2000 \mathrm{~K}$, whereas in Case 7, the medium temperature varies (somewhat arbitrarily) between $2100 \mathrm{~K}$ and $2700 \mathrm{~K}$. Figures 5 and 6 show the results from Case 6 and 7 respectively. Here we only presented the comparison of $\nabla \cdot Q$ with the exact solution and convergence rate plots for $\mathrm{MC}$ and $\mathrm{QMC}$.

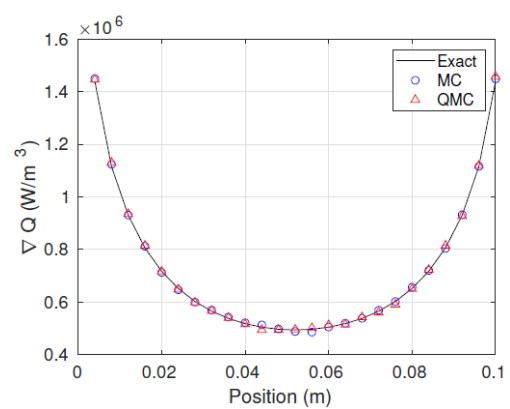

(a) Comparison of $\nabla \cdot Q$ with 100,000 rays

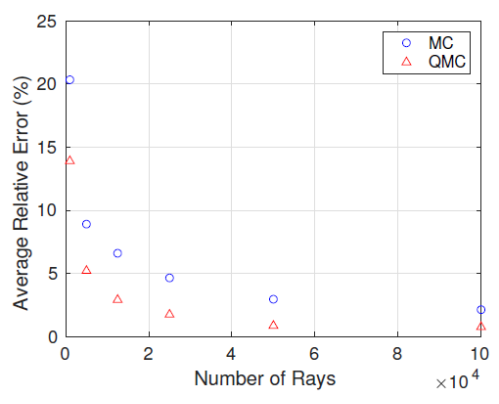

(b) Convergence rates

Fig. 5 Accuracy and convergence rates for Case 6 


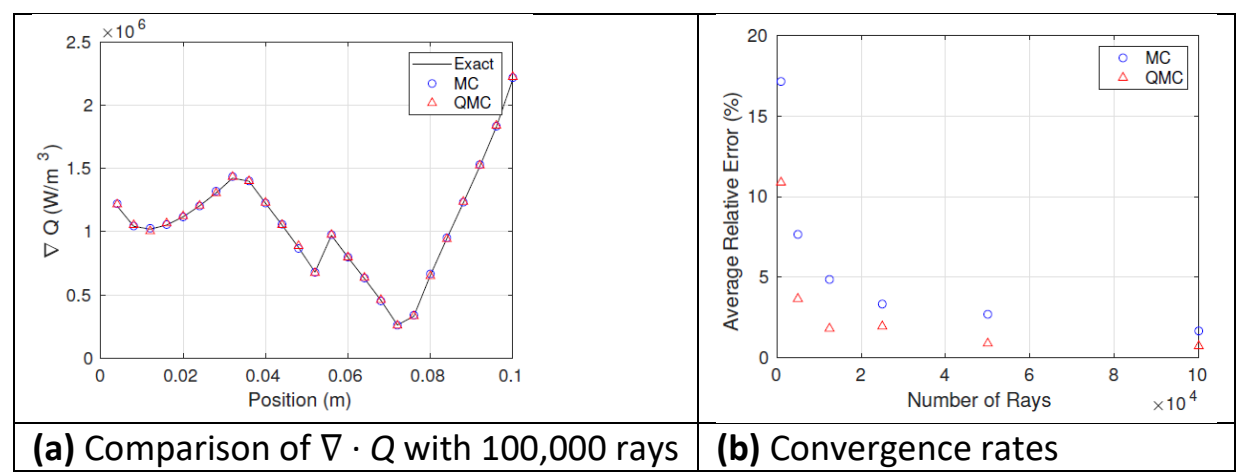

Fig. 6 Accuracy and convergence rates for Case 7

The trends seen in nongray cases are similar to that seen in gray cases. Both $\mathrm{MC}$ and $\mathrm{QMC}$ reproduce the exact solution within reasonable accuracy (Fig. 5a and Fig. 6a). Comparison of convergence rates (Fig. 5b and Fig. $6 \mathrm{~b}$ ) reveal that both $\mathrm{MC}$ and QMC converge to the exact solution at approximately the same rate, but for a given sample size (number of rays), error from QMC is significantly smaller than that from MC.

\subsection{Computational cost}

We also estimated the computational cost associated with both MC and QMC simulations. All the simulations were run on a single core of an Intel Xeon E3-1230v5 processor. Table 2 shows the computational time for one statistical run for $\mathrm{MC}$ and $\mathrm{QMC}$ for Case 1 using different number of rays. The computational cost of both solvers are comparable. The same trend is observed for all other cases (not shown here for brevity). At this point, we would like to highlight that for a conventional MC solver, it is often necessary to perform a "smoothing" by averaging over several statistical runs (e.g., 10 statistical runs used in this study), whereas for QMC just one single simulation is adequate because of the deterministic sampling.

Table 2 Execution times (s) for MC and QMC for Case 1

\begin{tabular}{|l|l|l|l|l|l|}
\hline 10000 Rays & & 5000 Rays & & 1000 Rays & \\
\hline MC & QMC & MC & QMC & MC & QMC \\
\hline 0.39 & 0.38 & 0.21 & 0.20 & 0.06 & 0.05 \\
\hline
\end{tabular}

\section{CONCLUSIONS}

This study shows a proof-of-concept for a quasi-Monte Carlo solver for radiation through participating media. Both $\mathrm{MC}$ and QMC solvers converge to the analytical solution. The results show clear advantages of QMC over standard MC simulations in plane-parallel gray and nongray medium. The advantage of low relative error from $\mathrm{QMC}$ is more prominent in optically thick regions than in optically thin regions. Computational cost of QMC is also comparable to MC. Combinations of lower error, deterministic sampling, and no additional computation overhead indicate that a fewer number of rays and only one statistical run may be needed in QMC to achieve similar levels of accuracy as the standard MC method in the studied configurations. Since the fundamental physics of the radiation in participating media does not change from configuration to configuration, it is expected that the advantages of QMC will also be evident in more complex radiative transfer problems in participating media. A detailed and systematic 
study of advantages of QMC is currently being explored in generic non-gray, three-dimensional problems.

\section{ACKNOWLEDGMENTS}

This work is supported by the National Science Foundation (award \# 1756005).

NOMENCLATURE

\begin{tabular}{|c|l|c|c|l|c|}
\hline$\kappa_{\eta}$ & $\begin{array}{l}\text { Absorption } \\
\text { Coefficient }\end{array}$ & $\left(\mathrm{cm}^{-1}\right)$ & $\sigma_{s \eta}$ & Scattering Coefficient & $\left(\mathrm{cm}^{-1}\right)$ \\
\hline$I_{b \eta}$ & Planck Function & $\left(\mathrm{Wcm}^{-2} \mathrm{sr}^{-1} \mu \mathrm{m}^{-1}\right)$ & $\hat{s}_{i}$ & Direction & $(-)$ \\
\hline$\beta_{\eta}$ & $\begin{array}{l}\text { Extinction } \\
\text { Coefficient }\end{array}$ & $\left(\mathrm{cm}^{-1}\right)$ & $\eta$ & Wavenumber & $\left(\mathrm{cm}^{-1}\right)$ \\
\hline$I_{\eta}$ & Radiative Intensity & $\left(\mathrm{Wcm}^{-2}\right)$ & $\Phi_{\eta}\left(\hat{s}_{i}, \hat{s}\right)$ & $\begin{array}{l}\text { Scattering Phase } \\
\text { Function }\end{array}$ & $(\mathrm{sr})$ \\
\hline$\nabla \cdot Q$ & $\begin{array}{l}\text { Divergence of Heat } \\
\text { Flux }\end{array}$ & $\left(\mathrm{Wm}^{-3}\right)$ & $i$ & Cell Number & $(-)$ \\
\hline$\varepsilon$ & Relative Error & $(-)$ & & & \\
\hline
\end{tabular}

\section{REFERENCES}

[1] M. Modest, Radiative Heat Transfer. Academic Press, 2013.

[2] J. Jeans, "The equations of radiative transfer of energy," Royal Astronomy Society, vol. 78, pp. 28-36, 1917.

[3] W. Ge, T. Ren, M. F. Modest, S. Roy, and D. C. Haworth, "Application of high-order spherical harmonics methods for radiative transfer in simulation of a turbulent jet flame," in Proceedings of CHT-17, ICHMT International Symposium on Advances in Computational Heat Transfer, (Napoli, Italy), 2017.

[4] W. Ge, M. F. Modest, and R. Marquez, "Two-dimensional axisymmetric formulation of high order spherical harmonics methods for radiative heat transfer," Journal of Quantitative Spectroscopy and Radiative Transfer, vol. 156, pp. 58-66, 2015.

[5] W. Ge, M. F. Modest, and S. P. Roy, "Development of high-order $P_{N}$ models for radiative heat transfer in special geometries and boundary conditions," Journal of Quantitative Spectroscopy and Radiative Transfer, vol. 172, pp. 98--109, 2015.

[6] M. F. Modest and J. Yang, "Elliptic PDE formulation and boundary conditions of the spherical harmonics method of arbitrary order for general three-dimensional geometries," Journal of Quantitative Spectroscopy and Radiative Transfer, vol. 109, no. 9, pp. 1641-1666, 2008.

[7] W. Ge, R. Marquez, M. F. Modest, and S. P. Roy, "Implementation of high-order spherical harmonics methods for radiative heat transfer on OpenFOAM," Journal of Heat Transfer, vol. 137, no. 5, p. 052701, 2015.

[8] S. Chandrasekhar, Radiative Heat Transfer. Dover Publications, 1960.

[9] J. C. Chai, H. S. Le, and S. V. Patankar, "Ray effect and false scattering in the discrete ordinates method," Numerical Heat Transfer, vol. 24, pp. 373-389, 1994.

[10] P. J. Coelho, "Discrete ordinates and finite volume methods," Thermopedia, 2011. http://www.thermopedia.com. 
[11] S. H. Kang and T. H. Song, "Finite element formulation of the first- and second-order discrete ordinates equations for radiative heat transfer calculation in three-dimensional participating media," Journal of Quantitative Spectroscopy and Radiative Transfer, vol. 109, no. 11, pp. 20942107, 2008.

[12] H. Hottel and E. Cohen, "Radiant heat exchange in a gas-filled enclosure: Allowance for nonuniformity of gas temperature," AIChE Journal, vol. 4, pp. 3-14, 1958.

[13] A. Eddington, The Internal Constitution of the Stars. Dover Publications, 1959.

[14] M. Modest and D. Haworth, Radiative Heat Transfer in Turbulent Combustion Systems. Springer, 2015.

[15] J. C. Chai, H. S. Lee, and S. V. Patankar, "Finite volume method for radiation heat transfer," Journal of Thermophysics and Heat Transfer, vol. 8, pp. 419-425, 1994.

[16] J. R. Howell, M. P. Menguc, and R. Siegel, Thermal Radiation Heat Transfer. CRC Press, 2015.

[17] S. Roy, J. Cai, W. Ge, and M. Modest, "Computational cost and accuracy comparison of radiation solvers with emphasis on combustion simulations," vol. 18 of 6, pp. 1493-1510, International Symposium on Advances in Computational Heat Transfer, 2015.

[18] G. Pal, A. Gupta, M. Modest, and D. Haworth, "Comparison of accuracy and computational expense of radiation models in simulation of non-premixed turbulent jet flames," Combustion and Flame, vol. 162, pp. 2487-2495, 2015.

[19] R. Ohbuchi and M. Aono, "Quasi-Monte Carlo rendering with adaptive sampling," tech. rep., IBM Tokyo Research Laboratory, 1996.

[20] D. M. O'Brien, "Accelarated quasi Monte Carlo integration of the radiative transfer equation," Journal of Quantitative Spectroscopy \& Radiative Transfer, vol. 48, pp. 41-59, 1992.

[21] A. Wang and M. F. Modest, "Spectral Monte Carlo models for nongray radiation analyses in inhomogeneous participating media," Int. J. Heat Mass Transfer, vol. 50, pp. 3877-3889, 2007.

[22] A. Wang and M. F. Modest, "Photon Monte Carlo simulation for radiative transfer in gaseous media represented by discrete particle fields," J. Heat Transfer, vol. 128, pp. 1041-1049, 2006.

[23] S. P. Roy, J. Cai, and M. F. Modest, "Development of a multiphase photon Monte Carlo method for spray combustion and its application in high-pressure conditions," International Journal of Heat and Mass Transfer, vol. 115, pp. 453-466, 2017.

[24] S. K. Park and K. W. Miller, "Random number generators: Good ones are hard to find," Communications of the ACM, vol. 31, pp. 1192-1201, Oct. 1988.

[25] H. Niederreiter, "Quasi-Monte Carlo methods and pseudo-random numbers," American Mathematical Society, vol. 84, pp. 957-1041, 1978.

[26] M. Giles, F. Kudo, I. Sloan, and B. Waterhouse, "Quasi-Monte Carlo for finance applications," Journal of Australian Mathematical Society, vol. 50, pp. 308-323, 2008.

[27] P. Brately and B. Fox, "Algorith 659: Implementing Sobol's quasirandom sequence generator," ACM Transactions on Mathematical Software, vol. 14, pp. 88-100, 1988.

[28] B. L. Fox, "Algorithm 647: Implementation and relative efficiency of quasirandom sequence generators," ACM Transactions on Mathematical Software, vol. 12, pp. 362-376, 1986.

[29] I. A. Antonov and V. M. Saleev, "An economic method of computing Ip - sequences," USSR Computational Mathematics and Mathematical Physics, vol. 19, pp. 252-256, 1979.

[30] S. Joe and F. Y. Kuo, "Remark on algorithm 659: Implementing Sobol's quasirandom sequence generator," ACM Transactions on Mathematical Software, vol. 29, pp. 49-57, 2003. 
[31] L. S. Rothman, I. E. Gordon, R. J. Barber, H. Dothe, R. R. Gamache, A. Goldman, V. I. Perevalov, S. A. Tashkun, and J. Tennyson, "HITEMP, the high-temperature molecular spectroscopic database," Journal of Quantitative Spectroscopy \& Radiative Transfer, vol. 111, no. 15, pp. 2139-2150, 2010. 